\title{
Subsidence of the Arctic stratosphere determined from thermal emission of hydrogen fluoride
}

\section{Citation}

Traub, Wesley A., Kenneth W. Jucks, David G. Johnson, and Kelly V. Chance. 1995. "Subsidence of the Arctic Stratosphere Determined from Thermal Emission of Hydrogen Fluoride." Journal of Geophysical Research 100 (D6): 11261. https://doi.org/10.1029/95jd00619.

\section{Permanent link}

http://nrs.harvard.edu/urn-3:HUL.InstRepos:41467438

\section{Terms of Use}

This article was downloaded from Harvard University's DASH repository, and is made available under the terms and conditions applicable to Other Posted Material, as set forth at http:// nrs.harvard.edu/urn-3:HUL.InstRepos:dash.current.terms-of-use\#LAA

\section{Share Your Story}

The Harvard community has made this article openly available.

Please share how this access benefits you. Submit a story.

Accessibility 


\title{
Subsidence of the Arctic stratosphere determined from thermal emission of hydrogen fluoride
}

\author{
Wesley A. Traub, Kenneth W. Jucks, David G. Johnson and Kelly V. Chance \\ Harvard-Smithsonian Center for Astrophysics, Cambridge, Massachusetts
}

\begin{abstract}
We determine subsidence in the Arctic stratospheric vortex from measurements of the column density of hydrogen fluoride, using a rotational line in thermal emission. The data were obtained with the far-infrared spectrometer on board the NASA DC-8 aircraft during the Arctic Airborne Stratospheric Expedition, from January through March 1992. A wide range of meteorological conditions was sampled, both inside and outside the polar vortex region. The measured values of subsidence are analyzed as a function of time of year and potential vorticity. From this analysis we draw the following conclusions: (1) Subsidence is strongly correlated with potential vorticity. However, there appear to be other factors, including meteorological conditions, which play a lesser role. (2) The gradient of subsidence with respect to potential vorticity is large and approximately constant across the vortex wall and is small elsewhere. The gradient of subsidence with respect to horizontal distance is dramatically large across the vortex wall. (3) There appears to be substantial descent in the early winter vortex, from the evidence of the observed subsidence in January and the assumption of zero subsidence at the fall equinox. (4) Descent continues to occur in late winter, with measured vertical velocities similar to that inferred for early winter. The average early and late winter vertical velocity at a representative altitude of $18 \mathrm{~km}$ is $0.052( \pm 0.013) \mathrm{cm} \mathrm{s}^{-1}$. (5) At the measured rate of subsidence the time required to flush the stratosphere by one scale height is about 6 months.
\end{abstract}




\section{Introduction}

The polar vortex in the winter stratosphere is driven by reduced solar heat input to the absorbing ozone layer, allowing the stratosphere to cool and its pressure to drop. In this picture, a polar low-pressure system develops, outside air starts to move poleward, and the Coriolis force deflects the air to rotate in the same sense as the Earth, i.e., cyclonically [e.g., Schoeberl et al., 1992]. As the cooling air sinks and compresses, it is replaced by high-altitude air moving in from lower latitudes. This vertical compression of air, with replacement at the top, produces a downward transformation of mixing-ratio profiles [Toon et al., 1992]. In this paper we use our measurements of thermal emission from stratospheric hydrogen fluoride (HF), coupled with a subsidence model, to determine vertical displacement in the Arctic vortex during the northern hemisphere winter of 1991-1992.

\section{Observations}

The measurements reported here were made during the Arctic Airborne Stratospheric Expedition (AASE II) in 1992, using our far-infrared spectrometer (FIRS-2) on board the NASA DC- 8 aircraft. The FIRS-2 instrument is the same one we have flown on balloon platforms since 1987 [e.g., Traub et al., 1991, 1992], with several adaptations, as follows: The spectrometer views the stratosphere through a side port in the aircraft, sequentially recording thermal emission spectra at elevation angles of $32^{\circ}, 16^{\circ}$, $8^{\circ}, 4^{\circ}, 2^{\circ}, 1^{\circ}$, and $0^{\circ}$, followed by calibration scans of a hot and a cold blackbody source. This 700-s cycle is repeated while the aircraft is at or near cruise altitude, except for brief periods when the Sun is close to the line of sight. The viewing angles are selected by a tilting mirror, driven by a stepper motor, and controlled by a shaft encoder referenced to the aircraft roll angle as determined by the onboard inertial navigation system. The infrared radiation is focused by a fixed telescope mirror, then fed to the spectrometer. These two feed mirrors are rigidly mounted to the spectrometer, and the entire assembly is floated with respect to the aircraft on pneumatic isolators, to reduce vibration and potential interference fringe contrast losses. The aircraft optical window is removed; because of the difficulty of providing a suitable infrared-transmitting window, a pressure-tight enclosure is placed around the input optics, up to the spectrometer's vacuum tank, where a small-diameter polyethylene window admits light into the spectrometer.

The data reported here were obtained on 13 flights of the DC-8, each lasting about 10 hours, distributed as follows: 165 observations from flights on UT dates January
$14,16,19,22$, and 24, 1992, centered at about UT day January 19.5; 112 observations from February 17, 20, and 22, centered on February 20.4; and 177 observations from March 10, 12, 14, 18, and 20, centered on March 15.3. About $10 \%$ of the original data were discarded because observing conditions were poor, i.e., the DC-8 was flying through signal-degrading clouds, or it was changing altitude rapidly. The range of latitudes each month is nominally $38^{\circ} \mathrm{N}$ (Moffett Field, California) to $90^{\circ} \mathrm{N}$, except for one flight in February to $15^{\circ} \mathrm{N}$ (Puerto Rico).

The aircraft pressure altitude is usually about $11 \mathrm{~km}$, with occasional periods at about 10 and $12 \mathrm{~km}$; this means that at poleward latitudes the aircraft is generally above the tropopause.

Since the DC-8 flights covered a substantial fraction of the winter season and sampled a wide range of latitudes and vortex conditions and since the FIRS-2 was operating most of the time that the aircraft was at cruise altitude, we anticipate that the observations discussed in this paper can be taken as being broadly representative of conditions in the entire Arctic polar vortex.

The FIRS-2 measures the thermal emission spectrum of the stratosphere against the cold background of space. Since the radiated power in an emission line is dependent upon the temperature, through the level population and Planck function, and since the temperature in the stratosphere is essentially independent of solar elevation in the short term, we find that long-lived species, such as HF, have spectra which are constant from day to night.

Following Toon et al. [1992] and Mankin et al. [1990], we use $\mathrm{HF}$ as a tracer of vertical motion in the stratosphere. There are three reasons for using this tracer. (1) HF is produced in the stratosphere, starting with photodissociation of chlorofluorocarbons and $\mathrm{SF}_{6}$ and so has a positive vertical gradient in volume mixing ratio (VMR). If an air column is displaced downward, with replacement at the top by air from neighboring columns, then the total column abundance of HF will increase. If the nominal VMR profile is known, then the amount of vertical motion can be quantified from measurements of the change in column abundance. (2) HF is expected to be chemically unreactive, so abundance changes can be attributed to dynamical rather than chemical activity [Brasseur and Solomon, 1984]. (3) HF has an easily measured emission line in the FIRS-2 farinfrared spectrum. A detailed discussion of the evidence linking the subsidence parameter with actual descent of the HF mixing ratio profile is presented by Toon et al. [1992]. 


\section{Data Analysis}

Our data reduction procedures for both balloon and aircraft spectra are presented in detail elsewhere [Johnson et al., 1995], so the present discussion is limited to the most pertinent steps. For HF we analyze spectra from the upper four elevation angles only, since spectra from the lower three angles have reduced sensitivity due to water vapor opacity in the HF region. The HF line is a rotational transition which lies at $163.936 \mathrm{~cm}^{-1}$. In the high-frequency wing of this line there is a weak hot-band line of water vapor; from an examination of our balloon spectra, which show this region at high signal-to-noise ratio and with reduced pressure broadening, the catalogued position of this line is found to be incorrect, so it is changed in our line list from 163.9602 to $163.9650 \mathrm{~cm}^{-1}$.

For each spectrum obtained along the flight path, the overhead atmosphere is modeled with a nine-layer model; input parameters include atmospheric pressure at aircraft altitude and the atmospheric temperature profile. All meteorological data were provided by the Goddard theory group [e.g., Schoeberl et al., 1992], from National Meteorological Center (NMC) data, in the form of a curtain file, i.e., as a function of latitude and longitude along the flight track.

The model VMR profile is taken to have a fixed amplitude but variable vertical scale. The atmosphere is assumed to contract by the factor $(1+s)$, where $s$ is independent of altitude but may vary with time; $s$ is the dimensionless "degree of subsidence" (hereafter "subsidence") parameter introduced by Toon et al. [1992]. In other words, we assume that for a given parcel of air the quantity $(1+s) z$ is conserved with respect to changes in latitude, longitude, and time. With this scale transformation the VMR becomes

$$
\operatorname{VMR}(z)=\operatorname{VMR}_{0}\left[(1+s) z_{0}\right]
$$

Here $\operatorname{VMR}_{0}\left(z_{0}\right)$ is a reference profile appropriate to midlatitude conditions, with time-trending species (e.g., HF) scaled to expected 1992 values. The reference profile was kindly supplied by G. Toon (private communication, 1993). The reference profile and the profile corresponding to $s=0.5$ are shown in Figure 1 .

For each spectrum we iteratively vary the subsidence until the theoretical spectrum matches the observed spectrum in the neighborhood of the HF line, using a least squares fitting procedure. This is roughly equivalent to fitting the HF column density along the line of sight by adjusting the model VMR profile. Both $\mathrm{HF}$ and $\mathrm{H}_{2} \mathrm{O}$ are scaled with the same value of subsidence; no attempt was made to adjust $\mathrm{H}_{2} \mathrm{O}$ independently, since the $\mathrm{H}_{2} \mathrm{O}$ line has little effect on the HF feature. The fitting program pro-

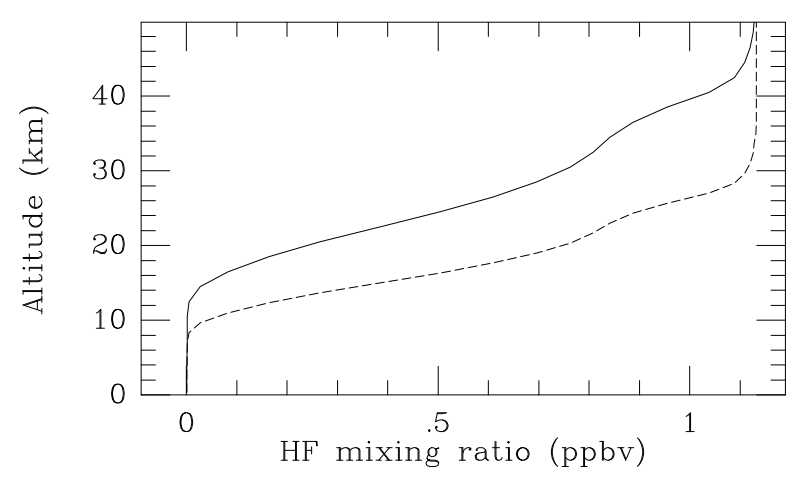

Figure 1. Reference mixing ratio profile for hydrogen fluoride (HF) (solid curve) and profile corresponding to a subsidence of 0.5 (dashed curve).

vides estimates for $s$ and the uncertainty $\delta s$. A weighted mean subsidence and uncertainty in the mean are calculated from the four independently determined values in each limb-scan sequence.

As mentioned above, wintertime cooling of the stratosphere causes the air pressure to decrease and the air to become denser, which in turn generates a cyclonic motion in the horizontal plane along with a sinking motion in the vertical direction. In this picture, stratospheric rotation and sinking are both driven by cooling, so in a simple model these effects are functionally related. In the actual atmosphere there are many competing mechanisms which will tend to degrade any straightforward functional relationship; but if rotation and sinking are the dominant effects, we should be able to observe a correlation between parameters which measure these motions.

Rotational motion is well characterized by the potential vorticity (PV) parameter [e.g., Brown, 1991; Peixoto and Oort, 1992], which is derived from satellite measurements of temperature and height. We use NMC values of potential vorticity, interpolated to the latitude, longitude, and time of our aircraft, and for an altitude corresponding to a potential temperature of $440 \mathrm{~K}$. The corresponding pressure altitude is about $18 \pm 1 \mathrm{~km}$, varying from about $17 \mathrm{~km}$ at midlatitudes to $19 \mathrm{~km}$ at high latitudes [e.g., Schoeberl et al., 1992]. This is roughly one scale height above the aircraft and also is close to the altitude of maximum sensitivity of the HF profile to vertical displacement (discussed below). For compactness we will write values in terms of the potential vorticity unit (PVU), defined here as

$$
\mathrm{PVU} \equiv 10^{-6} \mathrm{~K} \mathrm{~m}^{2} \mathrm{~kg}^{-1} \mathrm{~s}^{-1} \text {. }
$$

For adiabatic and frictionless motion, $\mathrm{PV}$ is a conserved quantity and should therefore serve as an indicator of dy- 


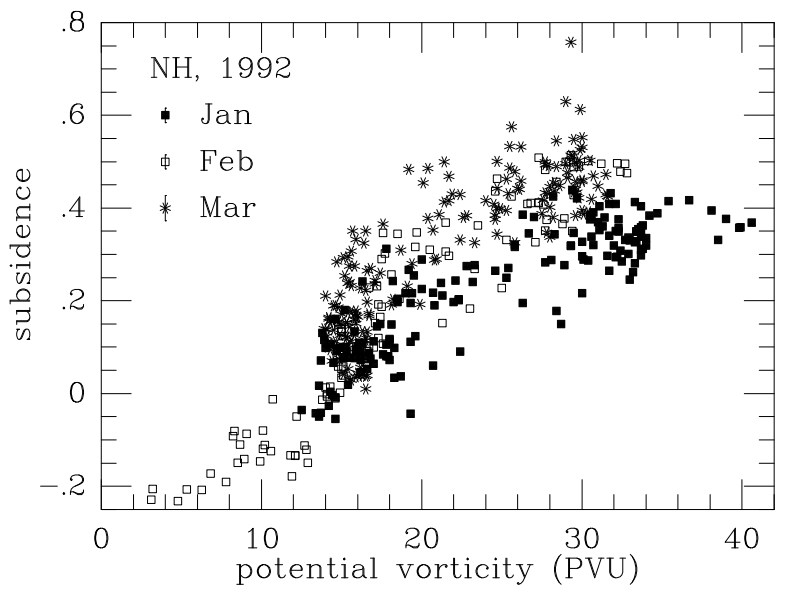

Figure 2. Stratospheric subsidence, as determined from DC- 8 based on observations of a far-infrared thermal emission line of HF, for the months of January, February, and March 1992, plotted against potential vorticity for the location and time of the aircraft. All data are from the northern hemisphere $(\mathrm{NH})$. Average uncertainties in individual determinations of subsidence are indicated in the legend.

namically similar parcels of air for as long as this assumption is valid. For periods longer than a week or so the effects of radiative heating and turbulence need to be considered [McIntyre and Palmer, 1983].

The derived values of subsidence are shown in Figure 2, plotted as a function of PV. The January, February, and March data are drawn as solid squares, open squares, and asterisks, respectively; the corresponding monthly-average uncertainties in individual subsidence values are 0.016, 0.015 , and 0.026 , indicated by vertical bars in the key to Figure 2. Each measurement averages over the area swept out by the field of view as the aircraft continues along the flight track. The along-track scale length of about $60 \mathrm{~km}$ is the distance traveled by the aircraft during a four-scan HF measurement; the cross-track scale length of about 45 $\mathrm{km}$ is the horizontal distance over which the line of sight rises by about one scale height. This area is small compared to the NMC analysis grid size of $2.5^{\circ}$ latitude by $5^{\circ}$ longitude.

There are several features of the groupings of points in Figure 2 which deserve mention. First, the scatter in the points appears greater than expected from the stated uncertainties. The evidence suggests that most of this scatter is due to different histories of the air masses, since for a given narrow range of PV values, there is significantly less scatter among subsidences that are closely adjacent than among those that are separated by large distances or times. A contributing factor may be small-scale variations in PV which are missed by the coarse grid used in the NMC analysis.

Second, some values of subsidence are likely shifted due to local meteorology, thus increasing the scatter. For example, the lowest January points between about 20 to 30 PVU are from the January 19 crossing of the North Atlantic, during which time a large tropospheric uplift occurred. Since the observed subsidences are weak, this uplift apparently affected at least the lower stratosphere as well.

Third, there are clusters of points near values of 16 and 30 PVU and relatively fewer points between these clusters. This is due to the extreme spatial steepness of the vortex wall, which tends to lie between values of about 19 and 30 PVU (see "gradients" subsection below). As the aircraft entered or exited the vortex, the wall was usually traversed quickly, although in some cases we did fly roughly parallel to the wall, and this accounts for more points being in the wall region than would be expected from a random sample of geographic points.

Fourth, a series of very low values of PV was encountered in February, when we flew unusually far south, from Maine to south of Puerto Rico. That these points exhibit negative subsidence (i.e., relative uplifting) is not unexpected, because the template HF profile was selected to be typical of more northerly latitudes.

\section{Subsidence Trends}

We search for trends in the data in Figure 2 using the following statistical procedure. We bin the data according to the values of two parameters: time and potential vorticity. The data from each month are binned in separate groups. Within each month, the data are binned by $\mathrm{PV}$. We can assume PV is conserved for each group since the observations during a given month span a period of not more than 10 days. We use equal-sized bins of width $\Delta \mathrm{PV}=2 \mathrm{PVU}$, on centers $\mathrm{PV}_{i}=i \mathrm{PVU}$, where $i=1-40$. With these choices, adjacent median values are correlated. Bins containing fewer than a threshold number of points are individually widened until they pass the threshold, here set to 6; this helps bridge data-poor regions.

For the $n_{i}$ points in each bin the median value $\bar{s}$ of subsidence is found. The uncertainty in each median value is calculated by assuming that the number of samples in each bin can fluctuate statistically by about $\Delta n_{i}=\sqrt{n_{i}}$. For an upper limit we calculate a perturbed median for the case in which the $\Delta n_{i}$ points having the smallest value of subsidence are removed from the sample; the lower limit is ob- 


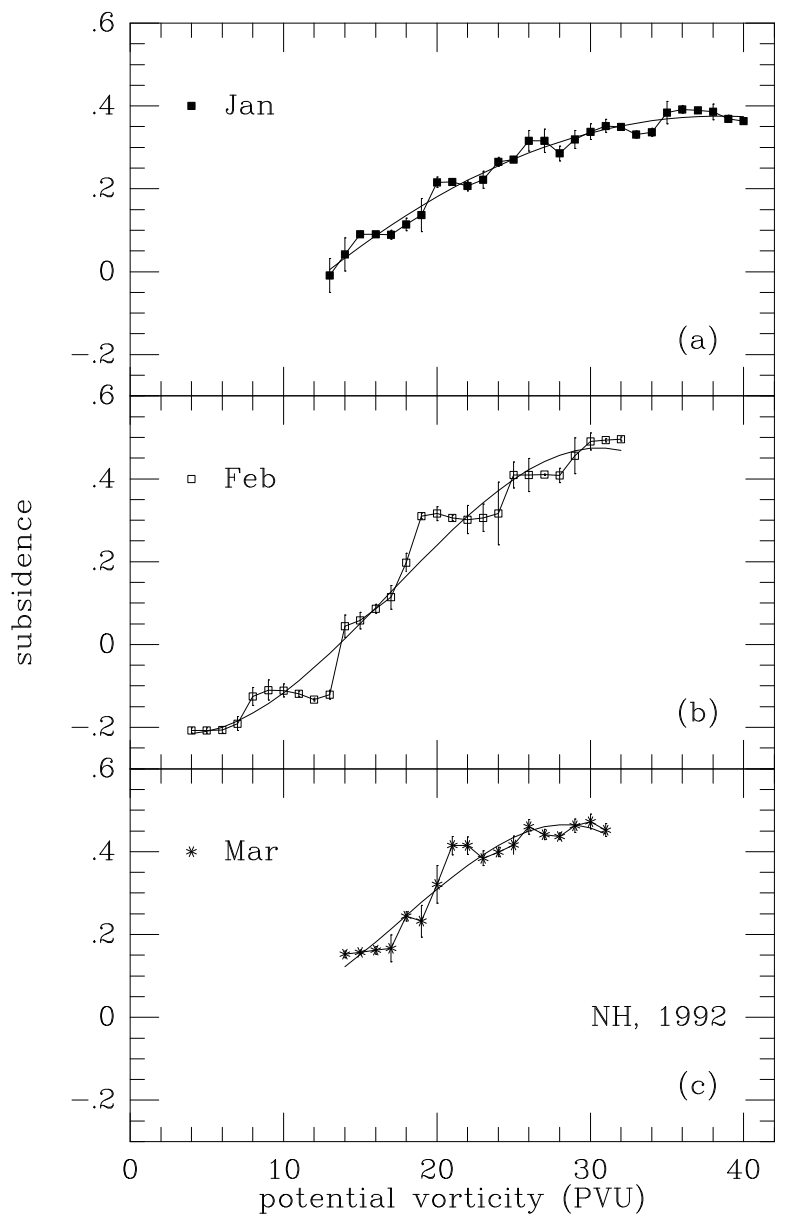

Figure 3. (a) Median values of subsidence are shown for January 1992 observations in bins of width $\triangle \mathrm{PV}=2 \mathrm{PVU}$, or greater (see text), spaced by intervals of one-half bin (1 PVU). The estimated uncertainty in each median value is indicated by a vertical bar. The smooth curve is that third-order polynomial $s(\mathrm{PV})$ which best fits the median values, in the sense of least squares. (b) Same, for February 1992. (c) Same, for March 1992.

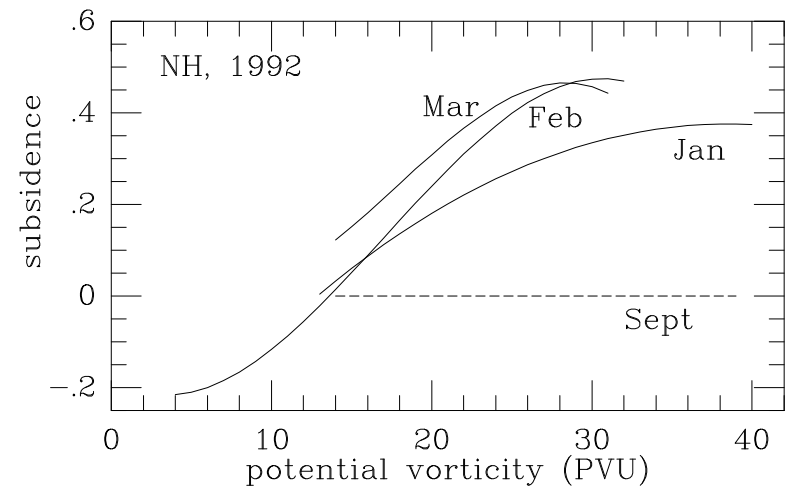

Figure 4. Polynomial curves $s(\mathrm{PV})$ for January, February, and March 1992, reproduced from Figure 3, showing the trend of stratospheric subsidence with respect to both PV and time. A dashed line indicates that zero subsidence is assumed to occur at about the fall equinox. For reference the vortex wall lies between PV values of $19 \pm 1 \mathrm{PVU}$ and $30 \pm 2 \mathrm{PVU}$ during all three months.

tained in a similar fashion. We expect that the uncertainties in the median are overestimated in bins where there are a large number of measurements but underestimated in bins where there happen to be a few clustered measurements; as such, the average uncertainty is probably more meaningful than any particular single value. The average uncertainties are $\delta s_{\text {ave }}=0.016,0.025$, and 0.019 for January, February, and March, respectively. The individual median values $\bar{s}$ and uncertainties $\delta \bar{s}$ are plotted in Figure 3.

Excluding the February flight to Puerto Rico, all median values have a lower limit of about 13 PVU, reflecting a common starting latitude at Moffett Field. The upper limits on median PV vary from 40 PVU in January to 31 PVU in March; this decrease with time possibly reflects a weakening of the vortex.

The dominant signal in the monthly median subsidence plots is a smooth trend of subsidence with respect to PV. To extract this trend, we construct a third-order polynomial function $s(\mathrm{PV})$ and perform a least squares fit to the median values $\bar{s}$, assuming equal weight at each point. These curves are shown superposed on the median values $\bar{s}$ in Figure 3 and, to facilitate comparison, are redrawn at the same scale in Figure 4.

In the remainder of this section we examine the data in Figures 2-4 for clues to the dynamical behavior of the polar vortex, taking advantage of the extended space-time coverage afforded by the DC- 8 flights. Conclusions are listed separately for clarity, although there is coupling between them. 


\section{Correlation With PV}

From the plots of median subsidence versus PV in Figure 3 , it is clear that there is a strong correlation between these quantities. The correlation is sufficiently good that a third-order polynomial can be passed through the data points and the curve taken to represent the data with little loss of information (even though neighboring points are correlated, fitting three parameters leaves 9-15 degrees of freedom). Quantitatively, the root-mean-square differences between the smooth curve $s$ and the median values $\bar{s}$ are $\delta s_{\text {rms }}=0.020,0.038$, and 0.034 for January, February, and March, respectively. These differences are about 1.5 and 1.7 times larger than the average uncertainties in the median and individual subsidences, respectively. This underscores the point made earlier that PV is not the sole determinant of subsidence and that there are other parameters, which we are ignoring here, which probably also play a role. In spite of this caveat concerning the role of secondary factors, Figure 3 clearly demonstrates that there is a strong correlation between the median subsidence and PV.

\section{Gradients}

In the range $\mathrm{PV} \simeq 13-30 \mathrm{PVU}$, Figure 3 shows that subsidence increases dramatically. For smaller values of $\mathrm{PV}$, toward the tropics, we expect that subsidence will become negative, reflecting a general upwelling driven by solar heating; however, in this case, the appropriate independent variable is likely to be latitude, not potential vorticity. For larger values of PV, Figure 3 suggests a saturation of subsidence, but there are too few data points here to uphold this speculation.

It is remarkable that the region of maximum slope $d s / d \mathrm{PV}$ coincides with the region of maximum slope of $\mathrm{PV}$ with respect to geographic distance $d \mathrm{PV} / d y_{\perp}$. Here $y_{\perp}$ indicates geographic distance in a direction perpendicular to contours of PV. Visual inspection of maps of PV [Tuck et al., 1992] illustrates the well-known fact that the polar vortex is bounded by a sharply defined "wall" where the contours of constant PV are closely bunched. The product of these gradients is the spatial gradient of subsidence,

$$
d s / d y_{\perp}=(d s / d \mathrm{PV}) \times\left(d \mathrm{PV} / d y_{\perp}\right) .
$$

Since both terms on the right-hand side of this equation are large at the location of the vortex wall (defined here as the region of maximum $d \mathrm{PV} / d y_{\perp}$ ), it follows that the spatial gradient of subsidence is particularly sharply defined there as well.

Our examination of PV maps for the days of observations reported here shows that the location of the wall in
PV space is the same each month. Measuring between points at the "bottom" and the "top" of the wall, corresponding visually to about the 10 and $90 \%$ amplitude contours, we find that the outer and inner edges are at

$$
\begin{aligned}
& \mathrm{PV}_{\text {out }} \simeq 19( \pm 1) \mathrm{PVU} \\
& \mathrm{PV}_{\text {in }} \simeq 30( \pm 2) \mathrm{PVU},
\end{aligned}
$$

respectively. The width of the wall is thus about

$$
\Delta_{\text {wall }} \mathrm{PV} \simeq 11( \pm 2) \mathrm{PVU}
$$

This corresponds to an Earth-centered width of

$$
\Delta_{\mathrm{wall}} \lambda \simeq 5.7( \pm 1.1)^{\circ}
$$

and a geographic distance of

$$
\Delta_{\text {wall }} \mathrm{Y}_{\perp} \simeq 640( \pm 120) \mathrm{km}
$$

Thus the transition region between inside and outside the vortex, or the subsided and nonsubsided stratosphere, occupies a narrow geographic band of width about $640 \mathrm{~km}$. By itself this does not say anything about where descent is taking place. To address this issue, we need to look at the change with time of the descended region, discussed in the next two subsections.

\section{Early Winter Subsidence}

The January trend line shows that by midwinter there was already a significant degree of subsidence in the vortex. Unfortunately, we do not have any measurements from earlier times, before the vortex was established. In addition to providing a baseline value of subsidence, these also would have been useful in estimating any time delay, or phase lag, between the onset of vorticity and the change of subsidence.

Despite the absence of such data, we may still attempt to estimate the vertical velocity during the first half of the winter season, by assuming that the prewinter subsidence is zero at the time of the fall equinox, in agreement with the value of subsidence we measure well outside the vortex in January. This assumption is consistent with the results of a two-dimensional model [Kaye et al., 1991] which shows almost no change with latitude in the HF column north of $50^{\circ}$ in September, whereas the column north of $60^{\circ}$ increases from September until the end of March (corresponding to an increase in subsidence for the same period). We assign an uncertainty of \pm 0.1 to the equinox subsidence. The zero level is indicated by a dashed line in Figure 4. 
We calculate the corresponding vertical displacement by noting that for a parcel of air the value of $(1+s) z$ is conserved, so taking differentials, we obtain

$$
\Delta z=-z \Delta s /(1+s),
$$

where $\Delta z$ is a small displacement about a mean value $z$ and $\Delta s$ is the change in subsidence about a mean value $s$. The vertical velocity $\bar{w}^{*}$ is calculated by dividing the displacement by the elapsed time $\Delta t$, so

$$
\bar{w}^{*}=\Delta z / \Delta t .
$$

In this notation the overbar indicates a time average and the superscript asterisk indicates a seasonal fluctuation with respect to the yearly average velocity (here assumed to be zero).

The change in subsidence from September to January is just the difference in the corresponding subsidence curves. The PV range over which one half or more of the peak subsidence occurs is 22-40 PVU, which we write as $31 \pm 9$ PVU. In this range, the average change in subsidence is $\Delta s=0.33 \pm 0.1$, and the time-average mean subsidence is $s=0.16$. At an altitude of $18 \mathrm{~km}$, which corresponds to the peak sensitivity of HF to descent [Toon et al., 1992] , we calculate a vertical displacement $\Delta z_{18}=-5.1( \pm 1.5) \mathrm{km}$. The relevant time interval is $\Delta t=120$ days, which then allows us to find the vertical velocity $\bar{w}^{*}=-0.049( \pm 0.015) \mathrm{cm} \mathrm{s}^{-1}$. These values are collected in Table 1, with the caveat that the initial condition is assumed, not measured.

\section{Late Winter Subsidence}

The greatest change in subsidence in the JanuaryFebruary interval is in a region spanned by $\mathrm{PV} \simeq$ $27( \pm 5) \mathrm{PVU}$, where the time-average subsidence is 0.36 and the increase is $0.12 \pm 0.02$. Following the above procedure, we find a vertical velocity $\bar{w}^{*}=-0.059( \pm 0.009)$ $\mathrm{cm} \mathrm{s}^{-1}$.

In comparing the measurements of subsidence for January and February, we are assuming that PV can be used as a conserved tracer for a month or more. While we do not expect PV to be strictly conserved for such a long period, as long as the change in PV is small, our estimate of the descent rate will not be affected. Our observations of the $\mathrm{HCl}$ column as a function of PV [Traub et al., 1994], binned and averaged for each month as is done for subsidence, are nearly identical for the months of January, February, and March. This indicates that we can assume that PV is conserved over longer periods when the data are averaged on a coarse grid as was done here.

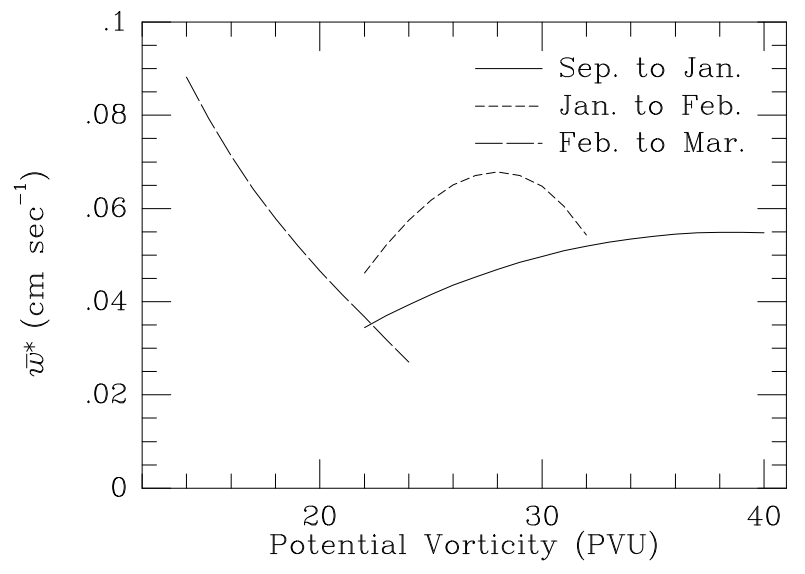

Figure 5. Derived vertical velocities for the periods September 1991 to January 1992, January to February 1992, and February to March 1992. The calculation of $\bar{w}^{*}$ is discussed in the text.

Likewise, for the period February to March, the greatest increase in subsidence occurred in the interval $\mathrm{PV} \simeq$ $19( \pm 5) \mathrm{PVU}$, where the average subsidence is 0.24 and the increase is $0.07 \pm 0.02$. The vertical velocity is -0.047 $\pm 0.014 \mathrm{~cm} \mathrm{~s}^{-1}$. These results also are in Table 1 .

It is interesting to note that the three derived vertical velocities are similar in both early and late winter; their average value is

$$
\bar{w}^{*}=-0.052( \pm 0.013) \mathrm{cm} \mathrm{s}^{-1} .
$$

This suggests that the air in the descending regions is continuing to cool at about the same rate. The derived vertical velocities for the ranges of PV being considered here are shown in Figure 5, calculated from the smoothed polynomial curves shown in Figure 4. At first glance, it may seem surprising that the stratosphere is still cooling as late as March, because the spring equinox occurs in that month and all latitudes up to the pole are in sunlight half of the time. However, it may be that what we are seeing here is simply the effect of a time constant in the stratospheric heating.

Our observed vertical velocities are marginally smaller than those estimated by Schoeberl et al. [1992] from calculated cooling rates in the north polar vortex region during the period January 3 to February 10, 1989. At an altitude of $18 \mathrm{~km}$ and a latitude around $60^{\circ} \mathrm{N}$, coincident with the polar jet and vortex edge, these authors find a zonal average vertical velocity of the order of $\bar{w}^{*}=-0.07 \mathrm{~cm} \mathrm{~s}^{-1}$. This is greater than our average measured value but not at a high level of significance. One might expect that the situation would be reversed, since a zonal average sam- 
Table 1. Stratospheric Subsidence From HF Observations

\begin{tabular}{cccccc}
\hline Period & $\begin{array}{c}\text { Interval } \\
\Delta t, \\
\text { days }\end{array}$ & $\begin{array}{c}\text { Subsiding } \\
\text { Region, } \\
\text { PVU }\end{array}$ & $\begin{array}{c}\text { Change in } \\
\text { Subsidence, } \\
\Delta s\end{array}$ & $\begin{array}{c}\text { Descent } \\
\Delta z_{18}, \\
\mathrm{~km}\end{array}$ & $\begin{array}{c}\text { Vertical } \\
\text { Velocity } \bar{w}^{*}, \\
\mathrm{~cm} \mathrm{~s}^{-1}\end{array}$ \\
\hline $\begin{array}{c}\text { Sept. 1991 to Jan. 1992 } \\
\text { Jan. to Feb. 1992 }\end{array}$ & 120 & $31 \pm 9$ & $0.33 \pm 0.10$ & $-5.1 \pm 1.5$ & $-0.049 \pm 0.015$ \\
Feb. to March 1992 & 32 & $27 \pm 5$ & $0.12 \pm 0.02$ & $-1.6 \pm 0.3$ & $-0.059 \pm 0.009$ \\
\hline
\end{tabular}

The potential vorticity unit (PVU) is defined in the text as $10^{-6} \mathrm{Km}^{2} \mathrm{~kg}^{-1} \mathrm{~s}^{-1}$.

ples many nonvortex locations and therefore should be a weaker measure.

\section{Time to Flush the Stratosphere}

From our measured winter-long average value of vertical velocity $\bar{w}^{*}$ and a nominal value of scale height $H$ of $8 \mathrm{~km}$, we can estimate the characteristic time to flush the stratosphere as

$$
T=H / \bar{w}^{*} \simeq 5.9 \pm 1.5 \text { month }
$$

at an altitude of about $18 \mathrm{~km}$. This suggests that the air in the stratosphere is replaced relatively slowly, of the order of only once per winter season.

\section{Location of Descent in PV Space}

From Figure 5 and the data in Table 1 the region of maximum descent velocity appears to move outward from the center of the vortex, from a position centered at $\mathrm{PV} \simeq 31 \mathrm{PVU}$ in early winter, to 27 followed by 19 in late winter. Because there is no reason to expect PV to be a conserved tracer for such a long period of time, we cannot determine whether this apparent shift is due to change in $\mathrm{PV}$ or in the region of descent without a more conservative tracer of motion.

\section{Conclusions}

From our observations of stratospheric HF in the polar vortex during the winter in 1992, we draw the following conclusions: (1) Subsidence is strongly correlated with potential vorticity. However, there appear to be other factors, including meteorological conditions, which play a lesser role. (2) The gradient of subsidence with respect to potential vorticity is large and approximately constant across the vortex wall and is small elsewhere. The gradient of subsidence with respect to horizontal distance is dramatically large across the vortex wall. (3) There appears to be substantial descent in the early winter vortex, from the evidence of the observed subsidence in January, and the assumption of zero subsidence at the fall equinox. (4) Descent continues to occur in late winter, with measured vertical velocities similar to that inferred for early winter. The average early and late winter vertical velocity at a representative altitude of $18 \mathrm{~km}$ is $0.052( \pm 0.013) \mathrm{cm} \mathrm{s}^{-1}$. (5) At the measured rate of subsidence, the time required to flush the stratosphere by one scale height is about 6 months.

Acknowledgments We thank Jim Changqin Xue for help in numerical calculations and the NASA Ames DC-8 flight crew for their superb support throughout the AASE II campaign. We are grateful to Mark Schoeberl, Leslie Lait, and Paul Newman for providing NMC curtain file data on temperature profiles and potential vorticity. This work was supported by NASA grant NSG 5175 .

\section{References}

Brasseur, G., and S. Solomon, Aeronomy of the Middle Atmosphere, D. Reidel, Norwell, Mass., 1984.

Brown, R. A., Fluid Mechanics of the Atmosphere, Academic, San Diego, Calif., 1991.

Johnson, D. G., K. W. Jucks, W. A. Traub, and K. V. Chance, Smithsonian stratospheric far-infrared spectrometer and data reduction system, J. Geophys. Res., 100, 3091-3106, 1995.

Kaye, J. A., A. R. Douglass, C. H. Jackman, R. S. Stolarski, R. Zander, and G. Roland, Two-dimensional model calculation of fluorine-containing reservoir species in the stratosphere, J. Geophys. Res., 96, 12,865-12,881, 1991. 
Mankin, W. G., M. T. Coffey, A. Goldman, M. R. Schoeberl, L. R. Lait, and P. A. Newman, Airborne measurements of stratospheric constituents over the Arctic in the winter of 1989, Geophys. Res. Lett., 17, 473-476, 1990.

McIntyre, M. E., and T. N. Palmer, Breaking planetary waves in the stratosphere, Nature, 305, 593-600, 1983.

Peixoto, J. P., and A. H. Oort, Physics of Climate, American Institute of Physics, New York, 1992.

Schoeberl, M. R., L. R. Lait, P. A. Newman, and J. E. Rosenfield, The structure of the polar vortex, J. Geophys. Res., 97, 7859-7882, 1992.

Toon, G. C., C. B. Farmer, P. W. Schaper, L. L. Lowes, R. H. Norton, M. R. Schoeberl, L. R. Lait, and P. A. Newman, Evidence for subsidence in the 1989 Arctic winter stratosphere from airborne infrared composition measurements, J. Geophys. Res., 97, 7963-7970, 1992.

Traub, W. A., K. V. Chance, D. G. Johnson, and K. W. Jucks, Stratospheric spectroscopy with the far-infrared spectrometer (FIRS-2): overview and recent results, SPIE, 1491, 298-307, 1991.

Traub, W. A., D. G. Johnson, K. W. Jucks, and K. V. Chance, Upper limit for stratospheric $\mathrm{HBr}$ using farinfrared thermal emission spectroscopy, Geophys. Res. Lett., 19, 1651-1654, 1992.

Traub, W. A., K. W. Jucks, D. G. Johnson, and K. V. Chance, Chemical change in the Arctic vortex during AASE II, Geophys. Res. Lett., 21, 2595-2598, 1994.

Tuck, A. F., et al., Polar stratospheric cloud processed air and potential vorticity in the northern hemisphere lower stratosphere at midlatitudes during winter, J. Geophys. Res., 97, 7883-7904, 1992.

This preprint was prepared with the AGU LATEX macros v3.1. File sub formatted 1999 March 12. 